\title{
Especificidade da armadilha Adultrap para capturar fêmeas de Aedes aegypti (Diptera: Culicidae)
}

\author{
Specificity of the Adultrap for capturing females \\ of Aedes aegypti (Diptera: Culicidae)
}

\author{
Almério de Castro Gomes ${ }^{1}$, Nilza Nunes da Silva ${ }^{1}$, Regina Tomie Ivata Bernal ${ }^{1}$, \\ André de Souza Leandro ${ }^{3}$, Natal Jataí de Camargo ${ }^{2}$, Allan Martins da Silva ${ }^{2}$, Adão Celestino Ferreira ${ }^{3}$, \\ Luis Carlos Ogura ${ }^{3}$, Sebastião José de Oliveira ${ }^{3}$ e Silvestre Marques de Moura ${ }^{3}$
}

\begin{abstract}
RESUMO
A Adultrap é uma nova armadilha feita para capturar fêmeas de Aedes aegypti. Foram realizados testes para avaliar sua especificidade tendo como referência a técnica da aspiração da espécie em abrigos artificiais. A Adultrap ficou exposta por 24 horas no intradomicílio e peridomicílio de 120 casas sorteadas em dois bairros da Cidade de Foz do Iguaçu, Estado do Paraná. O teste estatístico foi o modelo log-linear de Poisson. 0 resultado foi a captura de 726 mosquitos Culicidae, dos quais 80 eram Aedes aegypti. A Adultrap capturou apenas fêmeas desta espécie, enquanto o aspirador os dois sexos de Aedes aegypti e mais cinco outras espécies. A Adultrap capturou Aedes aegypti dentro e fora das casas, mas a análise indicou que no peridomicílio a armadilha capturou significantemente mais fêmeas do que a aspiração. Também, ficou evidenciada a sensibilidade da Adultrap para detectar Aedes aegypti em situação de baixa freqüência.
\end{abstract}

Palavras-chaves: Aedes aegypti. Vigilância. Armadilha para adulto.

\begin{abstract}
The Adultrap is a new trap built for capturing females of Aedes aegypti. Tests were carried out to evaluate the specificity of this trap in comparison with the technique of aspiration of specimens in artificial shelters. Adultraps were kept for 24 hours inside and outside 120 randomly selected bomes in two districts of the City of Foz do Iguaçú, State of Paraná. The statistical test was Poisson's log-linear model. The result was 726 mosquitoes captured, of which 80 were Aedes aegypti. The Adultrap captured only females of this species, while the aspiration method captured both sexes of Aedes aegypti and another five species. The Adultrap captured Aedes aegypti inside and outside the homes, but the analysis indicated that, outside the homes, this trap captured significantly more females than aspiration did. The sensitivity of the Adultrap for detecting females of Aedes aegypti in low-frequency situations was also demonstrated.
\end{abstract}

Key-words: Aedes aegypti. Surveillance. Adultrap.

A forma alada de Aedes aegypti dispersa-se por todo 0 ambiente domiciliar e tem ação direta na transmissão dos sorotipos do dengue, mas na medida da infestação urbana usase apenas o levantamento dos criadouros de larvas/pupas como ferramenta entomológica principal55.

As armadilhas freqüentemente consistem de combinações de atrativos que os mosquitos respondem, porém sabe-se que diferentes espécies não são igualmente atraídas pelos mesmos estímulos $^{8}$. Para Aedes aegypti existem inúmeras armadilhas para capturar adultos, obviamente cada uma com suas vantagens e desvantagens $^{2413}$.

Reconhecer a necessidade de um método para aperfeiçoamento de vigilância do dengue, particularmente em situação de baixo nível de infestação, é uma meta a ser alcançada. A armadilha de oviposição (ovitrampa) apresenta esta sensibilidade, mas é apenas preconizada para vigilância de áreas ainda não infestadas por Ae aegypti, além de requerer um laboratório de apoio ${ }^{10}$. 0 método isca humana, conhecido como o mais produtivo na captura de fêmeas de $A e$ aegypti $i^{12}$, tem restrição ética por expor o operador à infecção pelo vírus. Para superar essas dificuldades, foram elaboradas diferentes armadilhas que empregam vários tipos de estímulos atrativos para adultos de Ae aegypti e outros culicídeos, todavia, a opção com melhor resultado tem sido a aspiração realizada em abrigos domiciliares, ainda que este método capture quaisquer outros mosquitos alados em abrigos naturais e artificiais ${ }^{12}$. Barata e col $^{1}$ mostraram que a técnica aspiração é

\footnotetext{
1. Faculdade de Saúde Pública, Universidade de São Paulo, São Paulo, SP. 2. Centro de Saúde Ambiental, Secretaria de Estado da Saúde do Paraná, Curitiba, PR. 3. Centro de Controle de Zoonoses, Secretaria Municipal de Saúde de Foz do Iguaçu, Foz do Iguaçu, PR.

Endereço para correspondência: Dr. Almério de Castro Gomes. Faculdade de Saúde Pública/USP. Av. Dr. Arnaldo 715, 01246-904 São Paulo, SP.

Telefax: $55113081-2108$

e-mail agcastro@usp.br

Recebido para publicação em 7/8/2006

Aceito em 29/1/2007
} 
um método que permite uma boa avaliação da distribuição da população de Ae aegypti em área urbana. A Adultrap inserese neste contexto como uma nova opção para captura de adultos da espécie.

A nova armadilha foi usada para capturar alado de Ae aegypti (Adultrap), baseando-se na atração da fêmea para locais sombreados, objetos de cor e forma escurecida, preferencialmente próximo aos recipientes contendo água, simulando condição adequada para repouso ou abrigo, após a alimentação sanguínea e antes da oviposição. Testes para avaliar este princípio atrativo foram desenvolvidos em campo. Adicionalmente, há interesse em se conhecer a eficácia do método para nortear seu emprego operacional ou recomendá-lo nas medidas da infestação de $A e$. aegypti, sobretudo na avaliação do impacto das ações sobre 0 adulto.

Portanto, o objetivo deste relato é apresentar o resultado do estudo da especificidade da Adultrap para capturar formas adultas de $A e$ aegypti, em ambiente domiciliar urbano, tendo como parâmetro de referência o resultado da captura feita com o método aspiração.

\section{MATERIAL E MÉTODOS}

0 estudo foi realizado no município de Foz do Iguaçu situado no Estado do Paraná, cujo Índice de Infestação Predial estava, no máximo, em torno de 1,5\%. Foram escolhidos dois bairros e uma amostra total de 120 domicílios definida por sorteio sistemático realizado sobre o cadastro existente no Banco de Reconhecimento Geográfico da Prefeitura ${ }^{7}$.

A Adultrap tem forma cilíndrica e na extremidade superior é côncava, contendo um espaço para passagem do mosquito. A inferior é convexa. Lateralmente, é revestida por uma tela fina, a qual está ligada a um recipiente para colocar água ou outra isca atrativa. Internamente, existem dois compartimentos separados por um material transparente em formato de cones contendo um orifício de tamanho suficiente para passagem do mosquito adulto. 0 recipiente para a isca é separado das partes internas da armadilha por uma tela, sem qualquer chance de contato direto do mosquito com a isca usada (Figura 1). As armadilhas usadas foram instaladas dentro (intra) e nas imediações (peri) de cada um dos 120 domicílios, sendo 60 domicílios em novembro de 2004 e 60 em março de 2005. Foram usados como atrativo a água de torneira e água fenada a $10 \%$ divididas em igual número. Essas ficaram expostas por 24 horas ininterruptas e, posteriormente, transportadas para exame no laboratório, onde os mosquitos foram contados e identificados. 0 método aspiração foi aplicado nos mesmos locais após a retirada da Adultrap. 0 tempo da aspiração foi de 15 minutos e o puçá contendo o material aspirado foi levado ao laboratório e colocado em câmara de vapores de clorofórmio. Os mosquitos mortos foram separados com pinças, somados, verificada a presença de sangue e identificados.

Além dos resultados descritivos, a análise estatística considerou o número de fêmeas Ae aegypti capturadas como

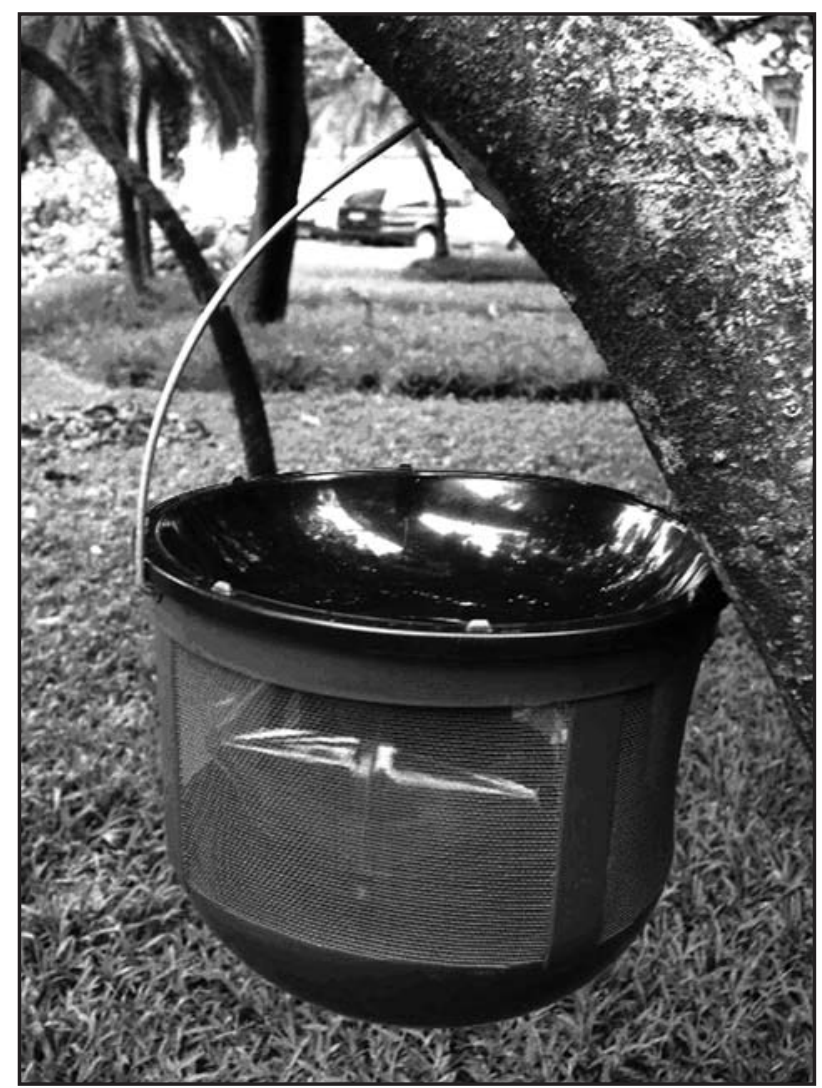

Figura 1 - Armadilba Adultrap.

a variável resposta, e usou o modelo log-linear de Poisson para avaliar os efeitos dos fatores estudados sobre sua distribuiçã̃ $0^{8}$.

0 modelo saturado nesse caso é dado por:

$$
\log \lambda_{\mathrm{ijk}}=\alpha+\beta_{\mathrm{i}}+\gamma_{\mathrm{j}}+\delta_{\mathrm{k}}+\eta_{\mathrm{j}}+\rho_{\mathrm{k}}
$$

em que $\beta_{\mathrm{i}}$ é o efeito da i-ésima armadilha Adultrap, $\gamma_{\mathrm{j}}$ é o efeito ao j-ésimo local peridomicílio, $\delta_{\mathrm{k}}$ é o efeito à k-ésima água fenada, $\eta_{\mathrm{ij}}$ denota a interação entre armadilha Adultrap e local peridomicílio e $\rho_{\mathrm{kj}}$ denota a interação entre à modificação de tipo da água fenada e local peridomicílio.

0 arquivo foi preparado segundo a perspectiva dicotômica desse modelo, em que a categoria 1 representava o tipo Adultrap para método de captura; o peridomicílio para instalação da armadilha; e a presença do feno para tipo de atração dos mosquitos.

\section{RESULTADOS}

A Tabela 1 mostra um total de 726 mosquitos capturados, dos quais 24 eram fêmeas de Ae aegypti; um Culex (Culex) spp e uma Coquillettia venezuelensis foram da Adultrap. 0 total da aspiração para Ae aegypti correspondeu a apenas 4\%. Os demais mosquitos capturados foram Culex quinquefasciatus e Aedes albopictus. A quantidade de Ae aegypti, de acordo com o número de fêmeas, local no domicílio e método de captura constam da Tabela 2. Os resultados estatísticos na primeira fase $\mathrm{da}$ modelagem, constantes da Tabela 3, apontaram que a presença do feno na água e o efeito de modificação do peridomicílio poderiam ser desconsiderados na aplicação do modelo. Nos resultados 
Tabela 1 - Quantidade de mosquitos capturados de acordo com a espécie, sexo, localização da armadilha, e método de captura.

\begin{tabular}{|c|c|c|c|c|c|c|c|c|c|c|c|c|}
\hline \multirow[b]{3}{*}{ Método } & \multirow[b]{3}{*}{ Local } & \multicolumn{10}{|c|}{ Espécies } & \multirow[b]{3}{*}{ Total } \\
\hline & & \multicolumn{2}{|c|}{ Ae aegypti } & \multicolumn{2}{|c|}{ Cx quinquefasciatus } & \multicolumn{2}{|c|}{$\mathrm{Cx} s p$} & \multicolumn{2}{|c|}{ Cq venezuelensis } & \multicolumn{2}{|c|}{ Ae albopictus } & \\
\hline & & fêmea & macho & fêmea & macho & fêmea & macho & fêmea & macho & fêmea & macho & \\
\hline & Intra & 2 & 0 & 0 & 0 & 0 & 1 & 1 & 0 & 0 & 0 & 4 \\
\hline \multirow[t]{3}{*}{ Adultrap } & Peri & 22 & 0 & 0 & 0 & 0 & 0 & 0 & 0 & 0 & 0 & 22 \\
\hline & Subtotal & 24 & 0 & 0 & 0 & 0 & 1 & 1 & 0 & 0 & 0 & 26 \\
\hline & Intra & 20 & 12 & 167 & 0 & 0 & 147 & 0 & 0 & 0 & 0 & 346 \\
\hline \multirow[t]{3}{*}{ Aspiração } & Peri & 9 & 15 & 115 & 2 & 0 & 212 & 0 & 0 & 1 & 0 & 354 \\
\hline & Subtotal & 29 & 27 & 282 & 2 & 0 & 359 & 0 & 0 & 1 & 0 & 700 \\
\hline & Total & 53 & 27 & 282 & 2 & 0 & 360 & 1 & 0 & 1 & 0 & 726 \\
\hline
\end{tabular}

Ae: Aedes; Cq: Coquillettia; Intra: intradomicílio; Peri: peridomicílio. Cx: culex.

Tabela 2 - Quantidade de Aedes aegypti de acordo o número de fêmeas capturadas, local no domicílio e método de captura.

\begin{tabular}{|c|c|c|c|c|c|c|c|c|}
\hline \multirow{3}{*}{$\begin{array}{l}\text { Número de fêmeas } \\
\text { capturadas }\end{array}$} & \multicolumn{4}{|c|}{ Adultrap } & \multicolumn{4}{|c|}{ Aspiração } \\
\hline & \multicolumn{2}{|c|}{ intra } & \multicolumn{2}{|c|}{ peri } & \multicolumn{2}{|c|}{ intra } & \multicolumn{2}{|c|}{ peri } \\
\hline & $\mathrm{n}^{\underline{0}}$ & $\%$ & $\mathrm{n}^{\underline{0}}$ & $\%$ & $\mathrm{n}^{\underline{0}}$ & $\%$ & $\mathrm{n}^{\underline{0}}$ & $\%$ \\
\hline 0 & 118 & 98,3 & 102 & 85,0 & 104 & 86,7 & 112 & 93,3 \\
\hline 1 & 2 & 1,7 & 16 & 13,3 & 13 & 10,8 & 7 & 5,8 \\
\hline 2 & - & - & 1 & 0,8 & 2 & 1,7 & 1 & 0,8 \\
\hline 3 & - & - & - & - & 1 & 0,8 & - & - \\
\hline 4 & - & - & 1 & 0,8 & - & - & - & - \\
\hline Total & 120 & 100,0 & 120 & 100,0 & 120 & 100,0 & 120 & 100,0 \\
\hline Taxas médias & \multicolumn{2}{|c|}{0,0167} & \multicolumn{2}{|c|}{0,1833} & \multicolumn{2}{|c|}{0,1667} & \multicolumn{2}{|c|}{0,0750} \\
\hline Variâncias & \multicolumn{2}{|c|}{0,0165} & \multicolumn{2}{|c|}{0,2686} & \multicolumn{2}{|c|}{0,2241} & \multicolumn{2}{|c|}{0,0868} \\
\hline
\end{tabular}

intra: intradomicílio; peri: peridomicílio

Tabela 3 - Modelo Log Poisson, estimativas e erro-padrão.

\begin{tabular}{|c|c|c|c|c|c|c|}
\hline \multirow[b]{2}{*}{ Efeito } & \multicolumn{3}{|c|}{ Primeira fase } & \multicolumn{3}{|c|}{ Segunda fase } \\
\hline & estimativa & erro-padrão & $\mathrm{P}$ & estimativa & erro-padrão & $\bar{P}$ \\
\hline Constante & $-1,791$ & 0,223 & 0,000 & $-1,791$ & 0,223 & 0,000 \\
\hline Adultrap & $-2,302$ & 1,024 & 0,025 & $-2,302$ & 1,024 & 0,025 \\
\hline Peridomicílio & $-0,708$ & 0,401 & 0,047 & $-0,798$ & 0,401 & 0,047 \\
\hline Água fenada & 0,559 & 0,443 & 0,207 & - & - & - \\
\hline Adultrap $p^{*}$ & 2,877 & 1,134 & 0,011 & 3,196 & 0,84 & 0,000 \\
\hline Água fenada* & $-0,559$ & 1,482 & 0,706 & - & - & \\
\hline
\end{tabular}

da segunda fase, nota-se que todos os efeitos são significativos ao nível de 5\%. Entretanto, os efeitos isolados da Adultrap e do peridomicílio são negativos, enquanto o efeito de modificação (Adultrap/peridomicílio) é positivo. Esse resultado levanta evidência de que a Adultrap aumentou o seu rendimento quando instalada no peridomicílio. Na amostra obtida, as fêmeas estavam alimentadas com sangue e até com ovos no abdome, enquanto na aspiração, tanto no intra quanto no peridomicílio, haviam fêmeas em jejum e com sangue.

Quanto ao sexo masculino, a Adultrap não capturou macho de Ae aegypti, enquanto a aspiração capturou macho de Ae aegypti tanto no intra (12) quanto no peridomicílio (15) e muitos machos de Cx. quinquefasciatus e Culex (Cux) spp.
Sendo assim, as duas técnicas apresentaram respostas diferentes quanto ao rendimento qualitativo e quantitativo.

\section{DISCUSSÃo}

As combinações de atrativos empregados pela Adultrap responderam a estímulos de abrigo para fêmeas de Ae aegypti. Nos ambientes investigados foram capturados, pelas duas técnicas, Ae aegypti e outras cinco espécies de culicídeos co-habitantes do intra e peridomicílio. Todavia, a força de atração da Adultrap para fêmeas de Ae aegypti sugere especificidade para este sexo do mosquito. Na amostra analisada não foi significativa a diferença entre água de torneira e água fenada, diferentemente do 
que foi demonstrado pela armadilha de oviposição $0^{11}$. Neste caso, parece que a armadilha atraia tanto grávidas quanto fêmeas em outros estágios de desenvolvimento folicular.

No que se refere ao rendimento da Adultrap, o resultado obtido no tempo de duração de 24 horas de captura, permite supor que, em período mais prolongado de exposição da mesma, exista a possibilidade de elevação do número de fêmeas a ser capturado. Isto também aumentaria a efetividade da armadilha no monitoramento dos impactos produzidos pelas medidas de controle sobre Ae aegypti.

A análise estatística mostrou que a Adultrap captura Ae aegypti tanto no intra como no peridomicílio das casas. Este local apresentou rendimento significativamente maior em relação ao aspirador. Num outro estudo quemediu a atividade das fêmeas com isca humana ${ }^{6}$, sob condição de baixa abundância, não foi assinalado diferença significante na atividade hematofágica entre 0 intra e peridomicílio, entendendo-se que uma vez as fêmeas alimentadas buscam abrigo fora dos domicílios. No Estado de São Paulo, a maioria dos criadouros da espécie em questão são encontrados no peridomicilio ${ }^{1}$, daí a dispersão das fêmeas alimentadas ao peridomicílio parecer ser um hábito freqüente, motivo pelo qual estas fizeram da Adultrap um dos abrigo procurados. Portanto, essas informações reforçam a suspeita da tendência da armadilha para capturar fêmeas em estado de repleção sanguínea e até em estágio de desenvolvimento folicular avançado ${ }^{2}$. Logo, parece que a aparente vantagem da Adultrap sobre a aspiração esteja na captura das fêmeas que estejam em vôo à procura de abrigo ou repouso.

Os machos não foram capturados pela Adultrap, sendo possível que estes orientem-se seus vôos para outros tipos de abrigos, ao contrário do aspirador que captura machos e fêmeas de mosquito em repouso no intra e peridomicílio ${ }^{3}$. Referindo-se ao estágio adulto mais conquistado pela armadilha, embora em número equivalente, Barata e $\mathrm{col}^{1}$ assinalaram, com aspirador, $82,5 \%$ dos machos no intradomicilio contra $17,6 \%$ no peridomicílio. Logo, a ausência deste sexo na Adultrap sugere especificidade da armadilha apenas para fêmeas.

Foi observado que a aspiração capturou mais culex quinquefasciatus do que Ae aegypti, evidenciando uma maior abundância da primeira espécie e uma baixa frequiência de adultos da segunda, nos domicílios pesquisados. Numa região do Peru $^{12}$, observou-se que a aspiração, para a coleta de mosquito, foi significantemente maior do que algumas armadilhas testadas, considerando assim este método como o mais efetivo para capturar Ae aegypti. No estudo de Foz do Iguaçu, a aspiração teve maior rendimento no intradomicílio, concordante com o estudo realizado em São José do Rio Preto ${ }^{1}$. Em ambas localidades, realizou-se intensa atividade de controle vetorial, sendo que na primeira o limite máximo do Índice de infestação Predial (IP) foi de $1,5 \%$. Conseqüentemente, supõe-se uma sensibilidade da Adultrap para detectar Ae aegypti, em períodos de baixa densidade, à semelhança do que ocorre com a ovitrampa ${ }^{10}$. Esta hipótese encontra respaldo na positividade da armadilha, sem encontro de criadouro da espécie nos domicílios.
Finalmente, a análise do rendimento da Adultrap configurase como potencial parâmetro de sensibilidade para avaliar a eficácia do controle vetorial, bem como na descrição dos níveis de transmissão do dengue. Operacionalmente, apresenta vantagem ao utilizar água de torneira para atrair Ae aegypti, fácil manipulação e ao mesmo tempo, ferramenta para estudos sobre a eficácia do método, da paridade e isolamento do vírus do dengue, por tratar-se de fêmeas alimentadas com sangue.

\section{AGRADECIMENTO}

Agradecemos às instituições Centro de Saúde Ambiental da Secretaria de Estado da Saúde do Paraná, Secretaria Municipal de Saúde de Foz de Iguaçú, pelo apoio ao desenvolvimento desse estudo.

\section{REFERÊNCIAS}

1. Barata EAM, Costa AIP, Chiaravalloti-Neto F, Glasser CM, Barata JMS, Natal D. População deAedes aegypti (I.) em área endêmica de dengue. Sudeste do Brasil. Revista de Saúde Pública 35:237-242, 2001.

2. Christophers SR. The development of egg folllicle in Anophelines. Paludism 2:73-89, 1911.

3. Clark GG, Seda H, Gubler DJ. Use of the "CDCbackback aspirator" for surveillance of Aedes aegypti in San Juan, Porto Rico. Journal American Mosquito Control Association 10:119-124, 1994.

4. Dennett JA, Vessey NY, Parsons RE. A comparison of seven traps used for collection of Aedes albopictus and Aedes aegypti originating from a large ire repository in Harris County (Houston), Texas. Journal American Mosquito Control Association 20:342-349, 2004.

5. Gomes AC. Medidas dos níveis de infestação urbana para Aedes (Stegomyia) aegypti e Aedes (Stegomyia) albopictus em programa de vigilância entomológica. Informe Epidemiológico do SUS 7: 49-57, 1998.

6. Gomes AC, Souza JMP, Bergamaschi DP, Andrade VR, Leite OF, Rangel O, Souza SSL, Guimarães NSN, Lima VLC. Atividade antropofílica de Aedes aegypti e Aedes albopictus em área sob controle e vigilância. Revista de Saúde Pública 39:206-210, 2005.

7. Kish L. longipalpis Survey sampling, John Wiley \& Sons, New York, 1965.

8. Lehtonen R, Pahlonen E. Practical methods for design and analysis of complex surveys. $2^{a}$ edition, John Wiley \& Sons, New York, 2004.

9. McCardle PW, Webb RE, Norden BB, Aldrich JR. Evaluation of five trapping systems for the surveillance of gravid mosquitoes in Prince Georges County, Maryland. Journal American Mosquito Control Association 20:254-260, 2004.

10. Morato VCG, Teixeira MG, Gomes AC, Bergamaschi DP, Barreto ML. Infestation of Aedes aegypti estimated by oviposition traps in Brazil. Revista de Saúde Pública 39: 553-558, 2005.

11. Reiter P, Amador MA, Colon N. Enhancement of the CDC ovitrap with hay infusions for daily monitoring of Aedes aegypti populations. Journal American Mosquito Control Association 7:52-55, 1991.

12. Schoeler GB, Schileich SS, Manweiler AS, Lopez-Sifuentes,V. Evaluation of surveillance devices for monitoring Aedes aegypti in an urban area of northeastern Peru. Journal American Mosquito Control Association 20:6-11, 2004

13. Trexler JD, Apperson CS, Gemeno C, Perich MJ, Carlson D, Schal C. Field and laboratory evaluation of potential oviposition attractans for Aedes albopictus (Diptera: Culicidae). Journal American Mosquito Control Association 19: 228-234, 2003 